\title{
Ferromagnetism in diluted magnetic semiconductor quantum dot arrays embedded in semiconductors
}

\author{
Pin Lyu ${ }^{1,2}$ and Kyungsun Moon ${ }^{1}$ \\ ${ }^{1}$ Department of Physics and Institute of Physics and Applied Physics, Yonsei University, Seoul 120-749, Korea \\ ${ }^{2}$ Department of Physics and Center for Theoretical Physics, Jilin University, Changchun 130023, China
}

(Dated: October 29, 2002)

\begin{abstract}
We present an Anderson-type model Hamiltonian with exchange coupling between the localized spins and the confined holes in the quantum dots to study the ferromagnetism in diluted magnetic semiconductor (DMS) quantum dot arrays embedded in semiconductors. The hybridization between the quantum-confined holes in the DMS quantum dots and the itinerant holes in the semiconductor valence band makes hole transfer between quantum dots, which can induce the long range ferromagnetic order of the localized spins. In addition, it makes the carrier spins both in the DMS quantum dots and in the semiconductors polarized. The spontaneous magnetization of the localized spins and the spin polarization of the holes are calculated using both the Weiss mean field approximation and the self-consistent spin wave approximation, which are developed for the present model.
\end{abstract}

PACS numbers: 75.75.+a, 75.30.Ds, 75.50.Dd, 75.50.Pp

\section{INTRODUCTION}

Diluted magnetic semiconductors (DMS) and a variety of quantum nanostructures based on DMS materials have recently attracted much interest due to the novel physics and the petential application to the emergent field of spintronics 1 The discovery of the ferromagnetism in the III-V based DMS materials $\mathrm{Ga}_{1-x} \mathrm{Mn}_{x} \mathrm{As}$ and $\mathrm{In}_{1-x} \mathrm{Mn}_{x}$ As has made it possible to combine the magnetic and semiconducting properties in one material.2 At high concentration of randomly distributed $\mathrm{Mn}^{2+}$ ions doped in GaAs samples with high hole density, $\mathrm{Ga}_{1-x} \mathrm{Mn}_{x}$ As compounds exhibit ferromagnetism with transition temperature as high as $110 \mathrm{~K}$ at certain value of $x$. The ferromagnetism in the localized spins of impurity $\mathrm{Mn}$ ions is mediated by the itinerant holes through the $p$ - $d$ exchange coupling brtirifen the valance-band holes and the localized spins 㔭地 Bhatt et al. argues that for low carrier density and strong disorder limit, the ferromagnetism is mediated by the carriers in impurity bands. 5 Recently the room temperature ferromagnetism was reported in the Mn-doped magnetic semiconductors $(\mathrm{Ga}, \mathrm{Mn}) \mathrm{N}$, which also belong to the III-V family.

As one of the interesting quantum structures made of the DMS materials, the self-organized (In,Mn)As quantum dots were successfully fabricated by growing (In,Mn)As on the (100), (211)B, (311)B GaAs substrates using low-temperature molecular beam epitaxy, which were subsequently capped by the GaAs layer. The electron diffraction pattern and the atomic force microscopy measurement confirmed the formation of the (In,Mn)As quantum dots. The more detailed experiments afterwards revealed that the majority of the Mn atoms were actually substituted in the In-site in (In,Mn)As quantum dots shown by the fluorescence extended x-rat absorption fine structure analysis $\mathrm{I}$ In their samples, $\mathrm{d}$ the (In, Mn)As quantum dots were irregularly placed and embedded in the GaAs. The major challenge in experi- ment is to fabricate the uniform and regular array of DMS quantum dots and to explore the various physical properties of these systems. The interplay between the quantum-confined magnetic dots and the non-magnetic semiconductors can be very interesting, which has a possible application as a tool to implement quantum bits, a large-scale quantum computer, 10 and other quantum devices.

In this paper, we theoretically study the ferromagnetism in diluted magnetic semiconductor quantum dot arrays embedded in semiconductors. In contrast to the bulk ferromagnetic semiconductors, the following two processes lead to the ferromagnetism in the present systems: (1) the localized spins of $\mathrm{Mn}^{2+}$ interact with the quantum-confined holes in the dots through exchange coupling, and (2) the localized orbital of the quantumconfined holes in the dots hybridizes with the itinerant holes in the semiconductor valence band. The hybridization allows hole transfer between the DMS quantum dots, which may induce the long range ferromagnetic order of the localized spins. In turn, the carrier spins both in the DMS quantum dots and in the semiconductors are polarized, which is crucial to realize spintronics. In order to describe the basic physics, we propose an Anderson-like model Hamiltonian, where the onsite Coulomb interaction is replaced by the exchange interaction. Our results provide a basis for the experiments on exploring the ferromagnetic properties of these systems.

The paper is organized as follows. In Sec. II, an Anderson-type model Hamiltonian is introduced to describe the DMS quantum dot arrays embedded in semiconductors. We use the Weiss mean field approximation and the self-consistent spin wave approximation, which are modified for the present model. In Sec. III, we show the numerical results of the temperature dependence of various physical quantities: the spontaneous magnetization of the localized spins and the spin polarizations of the carriers both in the quantum dots and in the semiconductor. Finally we conclude with a brief summary in 
Sec. IV.

\section{THEORETICAL MODEL AND SELF-CONSISTENT APPROXIMATION}

We consider the regular arrays of DMS quantum dots with the simple cubic structure embedded in the semiconductor such as $(\mathrm{Ga}, \mathrm{Mn}) \mathrm{As}$ or $(\mathrm{In}, \mathrm{Mn})$ As quantum dots in the GaAs Layer. The model Hamiltonian of the system can be written by

$$
\begin{aligned}
H= & \sum_{\mathbf{k} \sigma}\left(\varepsilon_{\mathbf{k}}-\mu\right) c_{\mathbf{k} \sigma}^{\dagger} c_{\mathbf{k} \sigma} \\
& +\sum_{i \sigma}\left(\varepsilon_{d}-\mu\right) d_{i \sigma}^{\dagger} d_{i \sigma}+J \sum_{i} \mathbf{S}_{i} \cdot \mathbf{s}_{i} \\
& +\sum_{i j \sigma} V \delta\left(\mathbf{R}_{i}-\mathbf{r}_{j}\right)\left(c_{j \sigma}^{\dagger} d_{i \sigma}+\text { H.c. }\right) .
\end{aligned}
$$

Here $c_{\mathbf{k} \sigma}$ and $d_{i \sigma}$ are the fermion operators for the carriers in the semiconductor and in the DMS quantum dots, respectively. For simplicity, we use the parabolic band for the carriers in the semiconductor $\varepsilon_{\mathbf{k}}=\hbar \mathbf{k}^{2} /\left(2 m^{*}\right)$ with the effective mass $m^{*}$. $\varepsilon_{d}$ is the discrete energy level of the carriers within the quantum dots, and $\mu$ is the chemical potential. The exchange coupling between the confined holes and $\mathrm{Mn}^{2+}$ ion impurity spins is special for the DMS quantum dot system and $J$ stands for the exchange coupling strength. $\mathbf{S}_{i}$ is the local spin of $\mathrm{Mn}$ ion impurity and $\mathbf{s}_{i}$ represents the spin of the confined holes at the $i$-th site of quantum dot arrays, which can be written as $\frac{1}{2} \sum_{\sigma \sigma^{\prime}} d_{i \sigma}^{\dagger} \vec{\tau}_{\sigma \sigma^{\prime}} d_{i \sigma^{\prime}}$, where $\vec{\tau}$ are the three Pauli spin matrices. $\mathbf{R}_{i}$ represents the $i$-th site of the DMS quantum dots in the arrays. The last term in the Hamiltonian (11) takes into account the hybridization between the holes confined in the quantum dots and the itinerant holes in the semiconductor valence band. The present Hamiltonian (11) is quite similar to that of the periodic Anderson model for the heavy fermion compounds 11 with the onsite screened Coulomb interaction. Here the onsite interaction is replaced by the exchange coupling between the localized spin and the hole confined in quantum dots.

Performing the coarse graining procedures 1 and using the Holstein-Primakoff transformations 12 the localized spin $\mathbf{S}_{i}$ can be written in terms of the bosonic operators $a_{i}^{\dagger}, a_{i}$ as follows

$$
\begin{gathered}
S_{i}^{z}=c S-a_{i}^{\dagger} a_{i}, \\
S_{i}^{+}=\sqrt{2 c S-a_{i}^{\dagger} a_{i}} a_{i}, \\
S_{i}^{-}=a_{i}^{\dagger} \sqrt{2 c S-a_{i}^{\dagger} a_{i}},
\end{gathered}
$$

where $c$ is the mean number of the magnetic ions $\mathrm{Mn}^{2+}$ in the DMS quantum dots. In the following, the approximation $\sqrt{2 c S-a_{i}^{\dagger} a_{i}} \simeq \sqrt{2 c S}$ is applied.
By transforming from the lattice space to the momentum space and limiting the involved momentum of the carriers in the semiconductor valence band within the first Brillouin zone of the corresponding quantum dot arrays, the Hamiltonian can be rewritten as follows,

$$
\begin{aligned}
H= & \sum_{\mathbf{k} \sigma}\left(\varepsilon_{\mathbf{k}}-\mu\right) c_{\mathbf{k} \sigma}^{\dagger} c_{\mathbf{k} \sigma}+\sum_{\mathbf{k} \sigma}\left(\varepsilon_{d \sigma}-\mu\right) d_{\mathbf{k} \sigma}^{\dagger} d_{\mathbf{k} \sigma} \\
& +\sum_{\mathbf{k} \sigma} V_{\mathrm{eff}}\left(c_{\mathbf{k} \sigma}^{\dagger} d_{\mathbf{k} \sigma}+\text { H.c. }\right) \\
& +\frac{J}{2} \frac{\sqrt{2 c S}}{\sqrt{M}} \sum_{\mathbf{q} \mathbf{k}}\left(a_{\mathbf{q}}^{\dagger} d_{\mathbf{k} \uparrow}^{\dagger} d_{\mathbf{k}+\mathbf{q} \downarrow}+a_{\mathbf{q}} d_{\mathbf{k}+\mathbf{q} \downarrow}^{\dagger} d_{\mathbf{k} \uparrow}\right) \\
& -\frac{J}{2 M} \sum_{\mathbf{k q}_{1} \mathbf{q}_{2} \sigma} \sigma a_{\mathbf{q}_{1}}^{\dagger} a_{\mathbf{q}_{2}} d_{\mathbf{k}-\mathbf{q}_{1} \sigma}^{\dagger} d_{\mathbf{k}-\mathbf{q}_{2} \sigma},
\end{aligned}
$$

where $\varepsilon_{d \sigma}=\varepsilon_{d}+\frac{\Delta}{2} \sigma$ with $\Delta=J c S, V_{\text {eff }}=V \sqrt{a^{3} / b^{3}}, b$ is the lattice constant of the quantum dot arrays, $a$ the effective radius of hybridization, and $M$ the total number of dot sites. The summations of $q, q_{1}$, and $q_{2}$ in the last two terms in the Hamiltonian (5) are restricted to the values less than the Debye cutoff $q_{c}$ for the spin waves of local impurity spins with the relation $q_{c}^{3}=6 \pi^{2} c / b^{3}$.

In functional integral representation, the partition function $Z$ for the Hamiltonian (5) is given by

$$
Z=\int \mathcal{D}\left[c^{\dagger} c\right] \mathcal{D}\left[d^{\dagger} d\right] \mathcal{D}\left[a^{\dagger} a\right] e^{-\int_{0}^{\beta} d \tau L\left(c^{\dagger} c, d^{\dagger} d, a^{\dagger} a\right)},
$$

where the Lagrangian $L$ can be written as

$$
\begin{aligned}
L= & \sum_{\mathbf{k} \sigma}\left(c_{\mathbf{k} \sigma}^{\dagger} \partial_{\tau} c_{\mathbf{k} \sigma}+d_{\mathbf{k} \sigma}^{\dagger} \partial_{\tau} d_{\mathbf{k} \sigma}\right)+\sum_{\mathbf{q}} a_{\mathbf{q}}^{\dagger} \partial_{\tau} a_{\mathbf{q}} \\
& +H\left(c^{\dagger} c, d^{\dagger} d, a^{\dagger} a\right) .
\end{aligned}
$$

Here the fermionic and bosonic degrees of freedom are represented by the Grassmann variables and the complex variables, respectively.

The itinerant carrier degrees of freedom in the semiconductor valence band can be integrated out easily, and we have

$$
Z=Z_{s} \int \mathcal{D}\left[d^{\dagger} d\right] \mathcal{D}\left[a^{\dagger} a\right] e^{-\int_{0}^{\beta} d \tau L\left(d^{\dagger} d, a^{\dagger} a\right)},
$$

where $Z_{s}$ is the partition function for the free carriers in the semiconductor valence band, and $L\left[d^{\dagger} d, a^{\dagger} a\right]$ is given by

$$
\begin{aligned}
L= & \sum_{\mathbf{k} \sigma} d_{\mathbf{k} \sigma}^{\dagger}\left[\partial_{\tau}-\mu+\varepsilon_{d \sigma}-V_{\mathrm{eff}}^{2}\left(\partial_{\tau}-\mu+\varepsilon_{\mathbf{k}}\right)^{-1}\right] d_{\mathbf{k} \sigma} \\
& +\sum_{\mathbf{q}} a_{\mathbf{q}}^{\dagger} \partial_{\tau} a_{\mathbf{q}}+\frac{J}{2} \frac{\sqrt{2 c S}}{\sqrt{M}} \sum_{\mathbf{q k}}\left(a_{\mathbf{q}}^{\dagger} d_{\mathbf{k} \uparrow}^{\dagger} d_{\mathbf{k}+\mathbf{q} \downarrow}\right. \\
& \left.+a_{\mathbf{q}} d_{\mathbf{k}+\mathbf{q} \downarrow}^{\dagger} d_{\mathbf{k} \uparrow}\right)-\frac{J}{2 M} \sum_{\mathbf{k} \mathbf{q}_{1} \mathbf{q}_{2} \sigma} \sigma a_{\mathbf{q}_{1}}^{\dagger} a_{\mathbf{q}_{2}} d_{\mathbf{k}-\mathbf{q}_{1} \sigma}^{\dagger} d_{\mathbf{k}-\mathbf{q}_{2} \sigma},
\end{aligned}
$$

where $\left(\partial_{\tau}-\mu+\varepsilon_{\mathbf{k} \sigma}\right)^{-1}$ is the Green's function of the itinerant carriers in the semiconductor valence band. 
By subsequently integrating out the remaining carrier degrees of freedom in the DMS quantum dots, we finally obtain the following partition function

$$
Z=Z_{s} \int \mathcal{D}\left[a^{\dagger} a\right] e^{-S_{\text {eff }},}
$$

where the effective action is given by

$$
S_{\text {eff }}=\int_{0}^{\beta} d \tau \sum_{\mathbf{q}} a_{\mathbf{q}}^{\dagger} \partial_{\tau} a_{\mathbf{q}}-\operatorname{Tr} \ln \left(G^{d}\right)^{-1}-\operatorname{Tr} \ln \left[\left(1+G^{d} \delta G^{-1}\right] .\right.
$$

The mean-field part $\left(G^{d}\right)^{-1}$ is written by

$$
\left(G^{d}\right)^{-1}=\left[\partial_{\tau}-\mu+\varepsilon_{d}-V_{\text {eff }}^{2}\left(\partial_{\tau}-\mu+\varepsilon_{\mathbf{k}}\right)^{-1}\right] \mathbf{1}+\frac{\Delta}{2} \tau^{z},
$$

and the fluctuation part $\delta G^{-1}$ is given by

$$
\begin{aligned}
\left\langle\mathbf{k}\left|\delta G^{-1}\right| \mathbf{k}^{\prime}\right\rangle= & \frac{J}{2} \frac{\sqrt{2 c S}}{\sqrt{M}}\left(a_{\mathbf{k}^{\prime}-\mathbf{k}}^{\dagger} \frac{\tau^{+}}{2}+a_{\mathbf{k}-\mathbf{k}^{\prime}} \frac{\tau^{-}}{2}\right) \\
& -\frac{J}{2 M} \sum_{\mathbf{q}} a_{\mathbf{q}-\mathbf{k}}^{\dagger} a_{\mathbf{q}-\mathbf{k}^{\prime}} \tau^{z} .
\end{aligned}
$$

Hence the exchange coupling and the hybridization of the carriers induce the effective non-local interaction between the magnetic impurities.

Expanding Eq. (11) to the quadratic order in $a^{\dagger}$ and $a$, we obtain

$$
S_{\mathrm{eff}}=\frac{1}{\beta} \sum_{\mathbf{q} i \nu_{m}} a_{\mathbf{q}}^{\dagger}\left(i \nu_{m}\right) D^{-1}\left(\mathbf{q}, i \nu_{m}\right) a_{\mathbf{q}}\left(i \nu_{m}\right)
$$

where the inverse of the spin wave propagator is given by

$$
\begin{aligned}
D^{-1}\left(\mathbf{q}, i \nu_{m}\right)= & -i \nu_{m}+\frac{J}{2 \beta M} \sum_{\mathbf{k} \sigma i \omega_{n}} \sigma G_{\sigma}^{d}\left(\mathbf{k}, i \omega_{n}\right) e^{i \omega_{n} 0^{+}} \\
& +\frac{J \Delta}{2 \beta M} \sum_{\mathbf{k} i \omega_{n}} G_{\uparrow}^{d}\left(\mathbf{k}, i \omega_{n}\right) \\
& \times G_{\downarrow}^{d}\left(\mathbf{k}+\mathbf{q}, i \omega_{n}+i \nu_{m}\right),
\end{aligned}
$$

and the Green function $G_{\sigma}^{d}\left(\mathbf{k}, i \omega_{n}\right)$ is written by

$$
G_{\sigma}^{d}\left(\mathbf{k}, i \omega_{n}\right)=\frac{-1}{i \omega_{n}-\left(\varepsilon_{d \sigma}-\mu\right)-V_{\mathrm{eff}}^{2} /\left[i \omega_{n}-\left(\varepsilon_{\mathbf{k}}-\mu\right)\right]}
$$

The spin wave dispersion is obtained by the following analytic continuation $i \nu_{m} \rightarrow \Omega+i 0^{+}$, which is given by

$$
\begin{aligned}
\Omega_{\mathbf{q}}= & \frac{J}{2 \beta M} \sum_{\mathbf{k} \sigma i \omega_{n}} \sigma G_{\sigma}^{d}\left(\mathbf{k}, i \omega_{n}\right) e^{i \omega_{n} 0^{+}}+\frac{J \Delta}{2 \beta M} \\
& \times \sum_{\mathbf{k} i \omega_{n}} G_{\uparrow}^{d}\left(\mathbf{k}, i \omega_{n}\right) G_{\downarrow}^{d}\left(\mathbf{k}+\mathbf{q}, i \omega_{n}+\Omega_{\mathbf{q}}\right) .
\end{aligned}
$$

After the Matsubara frequency summation, the spin wave dispersion can be derived as follows

$$
\Omega_{\mathbf{q}}=-\frac{J}{2 M} \sum_{\mathbf{k} \sigma \alpha} \sigma A_{\mathbf{k} \sigma}^{\alpha} f\left(\varepsilon_{\mathbf{k} \sigma}^{\alpha}\right)-\frac{J \Delta \lambda(\mathbf{q})}{2 M}
$$

$$
\begin{aligned}
& \times \sum_{\mathbf{k} \alpha}\left[A_{\mathbf{k} \uparrow}^{\alpha} A_{\mathbf{k}+\mathbf{q} \downarrow}^{\alpha} \frac{f\left(\varepsilon_{\mathbf{k}+\mathbf{q} \downarrow}^{\alpha}\right)-f\left(\varepsilon_{\mathbf{k} \uparrow}^{\alpha}\right)}{\Omega_{\mathbf{q}}+\varepsilon_{\mathbf{k} \uparrow}^{\alpha}-\varepsilon_{\mathbf{k}+\mathbf{q} \downarrow}^{\alpha}}\right. \\
& \left.+A_{\mathbf{k} \uparrow}^{\alpha} A_{\mathbf{k}+\mathbf{q} \downarrow}^{-\alpha} \frac{f\left(\varepsilon_{\mathbf{k}+\mathbf{q} \downarrow}^{-\alpha}\right)-f\left(\varepsilon_{\mathbf{k} \uparrow}^{\alpha}\right)}{\Omega_{\mathbf{q}}+\varepsilon_{\mathbf{k} \uparrow}^{\alpha}-\varepsilon_{\mathbf{k}+\mathbf{q} \downarrow}^{-\alpha}}\right]
\end{aligned}
$$

with $A_{\mathbf{k} \sigma}^{+}$and $A_{\mathbf{k} \sigma}^{-}$are given by

$$
A_{\mathbf{k} \sigma}^{+}=\frac{\varepsilon_{\mathbf{k} \sigma}^{+}-\varepsilon_{\mathbf{k}}+\mu}{\varepsilon_{\mathbf{k} \sigma}^{+}-\varepsilon_{\mathbf{k} \sigma}^{-}}
$$

and

$$
A_{\mathbf{k} \sigma}^{-}=\frac{\varepsilon_{\mathbf{k}}-\mu-\varepsilon_{\mathbf{k} \sigma}^{-}}{\varepsilon_{\mathbf{k} \sigma}^{+}-\varepsilon_{\mathbf{k} \sigma}^{-}}
$$

where the lower band energy is $\varepsilon_{\mathbf{k} \sigma}^{-}=\frac{1}{2}\left(\varepsilon_{d \sigma}+\varepsilon_{\mathbf{k}}\right)-$ $\frac{1}{2} \sqrt{\left(\varepsilon_{d \sigma}-\varepsilon_{\mathbf{k}}\right)^{2}+4 V_{\mathrm{eff}}^{2}}-\mu$ and the upper band $\varepsilon_{\mathbf{k} \sigma}^{+}=$ $\frac{1}{2}\left(\varepsilon_{d \sigma}+\varepsilon_{\mathbf{k}}\right)+\frac{1}{2} \sqrt{\left(\varepsilon_{d \sigma}-\varepsilon_{\mathbf{k}}\right)^{2}+4 V_{\mathrm{eff}}^{2}}-\mu$. The index $\alpha$ represents + or - for upper and lower hybridization bands, respectively and $f(x)$ is the Fermi-Dirac distribution function. If the last summation terms in Eq. (18) are ignored, we obtain the Weiss mean field approximation with the Weiss mean field given by $\Omega=$ $-J /(2 M) \sum_{\mathbf{k} \sigma \alpha} \sigma A_{\mathbf{k} \sigma}^{\alpha} f\left(\varepsilon_{\mathbf{k} \sigma}^{\alpha}\right)$, which is proportional to the spin polarization of the carriers in the DMS quantum dots. Here $\lambda(\mathbf{q})$ is introduced as a phenomenological renormalization factor in the last summation terms in Eq. (18), which effectively takes into account the higher order corrections in the fluctuations in $S^{x}$ and $S^{y}: \lambda(\mathbf{q})=\lambda_{0}+\left(1-\lambda_{0}\right) \tanh ^{2}\left(q / q_{c}\right)$. Since the Hamiltonian of the system is spin rotationally invariant and the ground state possesses a spontaneously broken symmetry, the system has a gapless Goldstone mode.12 The parameter $\lambda_{0}$ can be determined by imposing the following condition that $\Omega_{\mathbf{q}}=0$ as $\mathbf{q}$ goes to zero. At large values of $\mathbf{q}$, the renormalizations in the fluctuation term are negligible and so $\lambda(\mathbf{q})$ approaches to one. It appeared that for bulk ferromagnetic semiconductors, no gap arises in the self-consistent spin wave approximation, which we belipve is crucial to produce quantitatively reliable results 1 Hence the modification of the above approximation by putting in the renormlization factor $\lambda(\mathbf{q})$ can be very important for the present system.

At finite temperatures, the spin wave dispersion can be generalized by imposing the following self-consistency conditions for the finite temperature exchange gap $\Delta(T)$ given by $\Delta(T)=J_{p d}\left\langle S^{z}\right\rangle$. Here $\left\langle S^{z}\right\rangle$ represents the thermal average of the Mn ion spins in the DMS quantum dot arrays, which are approximately calculated by the following formulat

$$
\begin{aligned}
\left\langle S^{z}\right\rangle= & \frac{1}{M} \sum_{|\mathbf{q}|<q_{c}} S \mathcal{B}_{s}\left(\beta S \Omega_{\mathbf{q}}\right) \\
= & \frac{1}{M} \sum_{|\mathbf{q}|<q_{c}}\left\{S-n_{B}\left(\Omega_{\mathbf{q}}\right)\right. \\
& \left.+(2 S+1) n_{B}\left[(2 S+1) \Omega_{\mathbf{q}}\right]\right\},
\end{aligned}
$$


where $\mathcal{B}_{s}(x)$ is the Brillouin function and $n_{B}(x)$ the BoseEinstein distribution function. The second term in the second equality of Eq. (21) describes how the thermally induced spin waves from the independent Bose statistics with no limit in the number of spin waves reduce the magnetization of the system. The third term takes into account the correct spin kinematics, which rules out the unphysical states. When $\Omega_{\mathbf{q}}$ is independent of $\mathbf{q}$, for exmaple, the Weiss mean field approximation, Eq. (21) is reduced to the widely used formula $\left\langle S^{z}\right\rangle=c S_{\mathcal{B}}(\beta S \bar{\Omega})$.

On the other hand, using the Hamiltonian (5) without the exchange coupling, one can integrate out the carrier degrees of freedom in the DMS quantum dots to obtain the Green's function for the semiconductor carriers, where the role of the hybridization is taken into account. It is given by

$$
G_{\sigma}^{s}\left(\mathbf{k}, i \omega_{n}\right)=\frac{-1}{i \omega_{n}-\left(\varepsilon_{\mathbf{k}}-\mu\right)-V_{\mathrm{eff}}^{2} /\left[i \omega_{n}-\left(\varepsilon_{d \sigma}-\mu\right)\right]} .
$$

The densities of the carriers in the semiconductors and in the DMS quantum dots can be calculated from

$$
\begin{aligned}
& n_{\sigma}^{s}=\frac{1}{\pi \mathcal{V}} \sum_{\mathbf{k}} \int_{-\infty}^{\infty} d \omega f(\omega) \operatorname{Im} G_{\sigma}^{s}\left(\mathbf{k}, \omega+i 0^{+}\right), \\
& n_{\sigma}^{d}=\frac{1}{\pi \mathcal{V}} \sum_{\mathbf{k}} \int_{-\infty}^{\infty} d \omega f(\omega) \operatorname{Im} G_{\sigma}^{d}\left(\mathbf{k}, \omega+i 0^{+}\right),
\end{aligned}
$$

where $\mathcal{V}$ is the volume of the system. The total carrier density is given by $n_{\text {tot }}=\sum_{\sigma}\left(n_{\sigma}^{s}+n_{\sigma}^{d}\right)$, which is fixed and determines the Fermi energy.

The spin polarization of the carriers is defined as $P_{s}=$ $\left(n_{\downarrow}^{s}-n_{\uparrow}^{s}\right) /\left(n_{\downarrow}^{s}+n_{\uparrow}^{s}\right)$ in the semiconductor and $P_{d}=\left(n_{\downarrow}^{d}-\right.$ $\left.n_{\uparrow}^{d}\right) /\left(n_{\downarrow}^{d}+n_{\uparrow}^{d}\right)$ in the DMS quantum dots. This definition of spin polarizations is different from those commonly used in the transport properties, which is defined as the corresponding density of states at the Fermi energy 13

Based on the above framework, one can calculate the spontaneous magnetization of the localized spins in the DMS quantum dot arrays and the spin polarizations of the carriers both in the DMS quantum dots and in the semiconductor by solving the set of coupled equations in Weiss mean field approximation and self-consistent spin wave approximations as well.

\section{RESULTS AND DISCUSSIONS}

We have chosen the typical material parameters of the bulk (Ga,Mn)As for the DMS quantum dots, i.e. $J=0.15 \mathrm{eV}, m^{*}=0.5 m_{e}$. The lattice distance $b$ of the DMS quantum dots is set to be $b=1.0 \mathrm{~nm}$, which is within the spin-coherence length in semiconductor GaAs. 14 The carrier bands consist of the upper $\varepsilon_{\mathbf{k} \sigma}^{+}$ and lower $\varepsilon_{\mathbf{k} \sigma}^{-}$bands due to the hybridization between the discrete energy level of DMS quantum dot and the

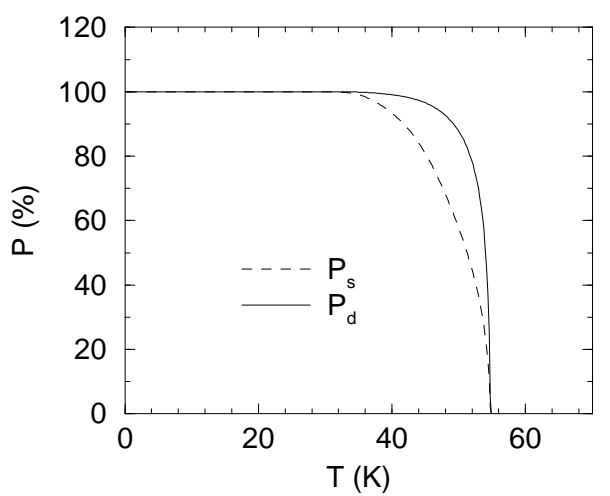

FIG. 1: Spin polarization of the carriers in DMS quantum dots $\left(P_{d}\right)$ and in semiconductors $\left(P_{s}\right)$ in Weiss mean field theory with the following parameters $J=0.15 \mathrm{eV}, m^{*}=$ $0.5 m_{e}, c^{*}=0.1 \mathrm{~nm}^{-3}, c=1.0, b=1.0 \mathrm{~nm}, V_{\text {eff }}=0.30 \mathrm{eV}$, and $\varepsilon_{d}=0.46 \mathrm{eV}$.

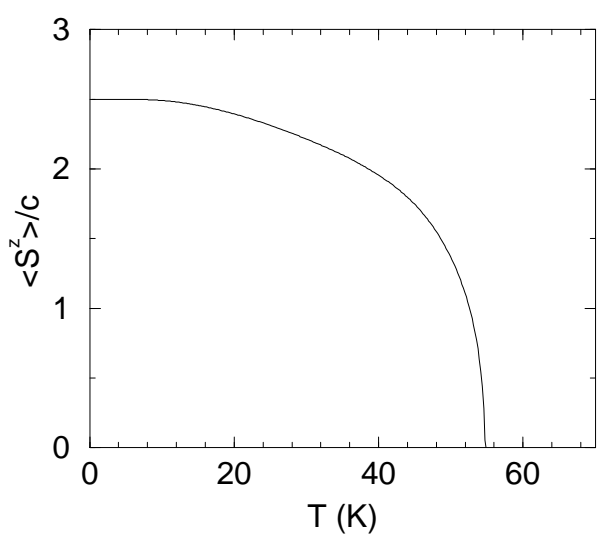

FIG. 2: Spontaneous magnetization of the localized spins in DMS quantum dot arrays in Weiss mean field theory with the same parameters used in Fig. 1.

semiconductor valence band. The spin dependence of the upper and lower bands is originated from the exchange coupling in the DMS quantum dots. Also there exists a gap between the spin-up (spin-down) upper and lower bands. When the ground state is metallic or semiconducting, the Fermi level must lie in the spin-down lower band.

In the following we calculate the ferromagnetic properties of the present system both in the Weiss mean field approximation and in the self-consistent spin wave approximation, respectively.

In Fig. 1 and Fig. 2, we plot the temperature dependence of the spin polarizations of the carriers in the semiconductor and in the DMS quantum dots, and the spontaneous magnetization for the DMS quantum dot arrays in the Weiss mean field theory with the following material parameters $c^{*}=0.1 \mathrm{~nm}^{-3}, c=1.0, V_{\text {eff }}=0.30 \mathrm{eV}$, and $\varepsilon_{d}=0.46 \mathrm{eV}$. The critical temperature $T_{c}$ for spin polarization is about $55 \mathrm{~K}$. One can notice that the spin polarizations $P_{d}$ and $P_{s}$ decrease more stiffly than the 


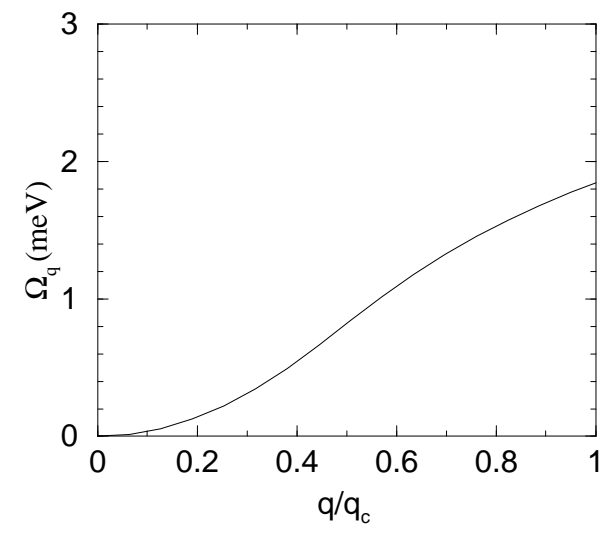

FIG. 3: Renormalized spin wave dispersion at $4.2 \mathrm{~K}$ in the self-consistent spin wave approximation with the same parameters used in Fig. 1.

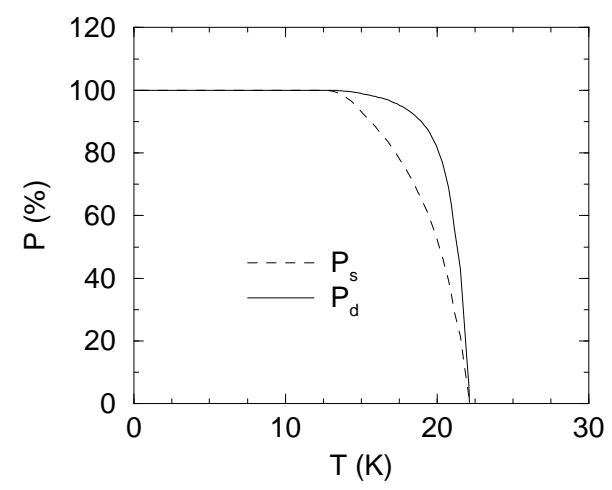

FIG. 4: Spin polarization of the carriers in DMS quantum dots $\left(P_{d}\right)$ and in semiconductors $\left(P_{s}\right)$ in the self-consistent spin wave approximation with the same parameters used in Fig. 1.

spontaneous magnetization $\left\langle S^{z}\right\rangle$ of localized spins. The $T_{c}$ presumably increases up to the bulk value as one tunes the exchange coupling strength $J$ and the hybridization $V_{\text {eff }}$ to higher values.

Next we go beyond the standard Weiss mean field theory. In Fig. 3, we have shown the renormalized spin wave dispersion curve at $T=4.2 \mathrm{~K}$ based on the the self-consistent spin wave approximation. The material parameters are the same as those used in the Weiss mean field calculation. The spin wave frequencies are less than the Weiss mean-field value $\Omega \simeq 4.14 \mathrm{meV}$. As q goes to zero, $\Omega_{\mathbf{q}} \propto q^{2}$, which are correct for ferromagnetic spin wave dispersion. As $\mathbf{q}$ becomes very large, $\Omega_{\mathbf{q}}$ approaches to the mean field value. Fig. 4 and Fig. 5 illustrate the temperature dependence of the spin polarizations of the carriers in the semiconductor and in the DMS quantum dots, and the spontaneous magnetization for the DMS quantum dot arrays in the self-consistent spin wave approximation with the same material parameters as before. The magnetization and the spin polarizations in the self-consistent spin wave approximations drop much rapidly comparing to those in the Weiss mean field ap-

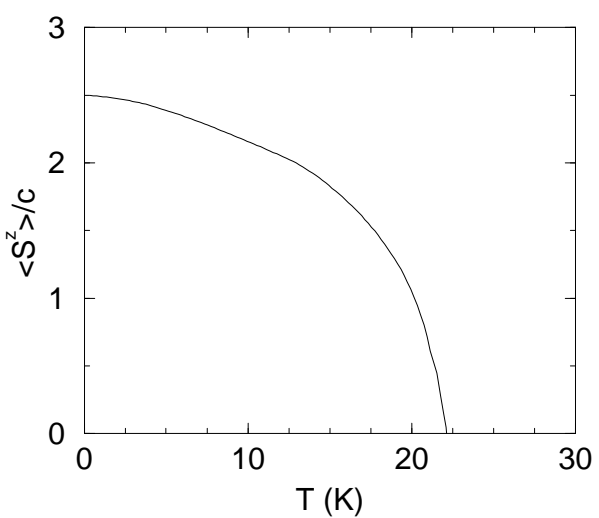

FIG. 5: Spontaneous magnetization of the localized spins in DMS quantum dot arrays in the self-consistent spin wave approximation with the same parameters used in Fig. 1.

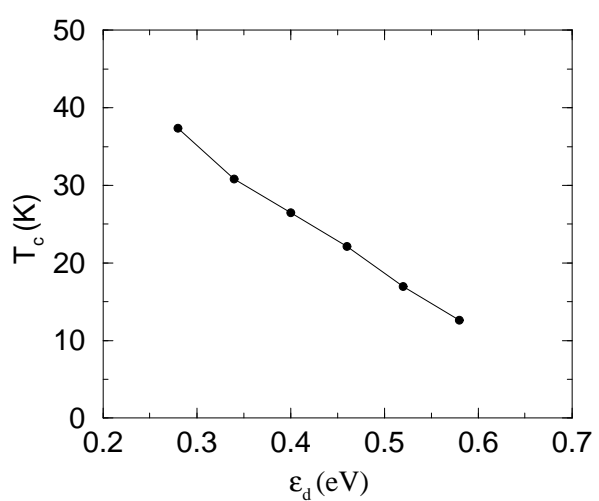

FIG. 6: Transition temperature $T_{c}$ versus discrete energy level $\varepsilon_{d}$ in the self-consistent spin wave approximation with the following parameters $J=0.15 \mathrm{eV}, m^{*}=0.5 m_{e}, c^{*}=$ $0.1 \mathrm{~nm}^{-3}, c=1.0, b=1.0 \mathrm{~nm}$, and $V_{\text {eff }}=0.30 \mathrm{eV}$.

proximation due to the strong spin fluctuations. The $T_{c}$ drops to about $22 \mathrm{~K}$ less than a half of the mean field value of $T_{c}$, which also happens in the bulk ferromagnetic semiconductors. 1 The qualitative behaviors remain the same as the mean field results.

Fig. 6 shows the dependence of the critical temperature $T_{c}$ as a function of the discrete energy level $\varepsilon_{d}$ of quantum dot. The $T_{c}$ increases with the decrease of $\varepsilon_{d}$. As $\varepsilon_{d}$ decreases, the carriers in the semiconductor easily hop to the localized levels and vice versa, which subsequently enhances the ferromagnetic coupling between impurity spins. The monotonic dependence of $T_{c}$ on $\varepsilon_{d}$ is only correct in the low carrier density region, in which our model is suitable, that is, the Fermi energy level lying in the spin-down lower band. In the low carrier density limit, the Fermi energy level always lies below $\varepsilon_{d \downarrow}$. Obviously the enhancement of the hybridization strength between the localized carriers in DMS quantum dots and the itinerant carriers in semiconductor makes carrier transfer easier leading to the increase of $T_{c}$. 


\section{SUMMARY}

We have theoretically studied the origin of the ferromagnetism in diluted magnetic semiconductor quantum dot arrays embedded in semiconductors based on an Anderson-type model Hamiltonian. The hybridization between the quantum-confined holes in the DMS quantum dots and the itinerant holes in the semiconductor valence band makes the hole transfer between the DMS quantum dots, which induces the long range ferromagnetic order of the localized spins in the DMS quantum dot arrays through the exchange coupling. Currently the available DMS quantum dot systems have irregular nanostructures, and the uniform and regular arrays of
DMS quantum dots are expected in experiments. Our results provide a basis for exploring the magnetic properties of these systems.

\section{ACKNOWLEDGMENTS}

It is our great pleasure to acknowledge useful discussions with R. N. Bhatt and T. K. Lee. This work was supported by the Brain Korea 21 Project and Grant No. R01-1999-00018 from the interdisciplinary Research program of the KOSEF. P. L. was also supported by SRF for ROCS from SEM of China and Research Foundation of Jilin University.
1 S. A. Wolf, D. D. Awschalom, R. A. Buhrman, J. M. Daughton, S. von Molnar, M. L. Roukes, A. Y. Chtchelkanova, and D. M. Treger, Science 294, 1488 (2001).

2 H. Ohno, Science 281, 951 (1998); J. Magn. Magn. Mater. 200, 110 (1999).

3 T. Dietl, H. Ohno, F. Matsukura, J. Cibert, and D. Ferrand, Science 287, 1019 (2000).

4 J. König, H.-H. Lin, and A. H. MacDonald, Phys. Rev. Lett. 84, 5628 (2000); J. König, T. Jungwirth, and A. H. MacDonald, Phys. Rev. B 64, 184423 (2001).

5 M. Berciu and R. N. Bhatt, Phys. Rev. Lett. 87, 107203 (2001); R. N. Bhatt, M. Berciu, M. P. Kennett, and X. Wan, J. Supercond. 15, 71 (2002).

${ }^{6}$ M. L. Reed, M. K. Ritums, H. H. Stadelmaier, M. J. Reed, C. A. Parker, S. M. Bedair, and N. A. El-Masry, Mater. Lett. 51, 500 (2001); M. L. Reed, N. A. El-Masry, H. H. Stadelmaier, M. K. Ritums, M. J. Reed, C. A. Parker, J. C. Roberts, and S. M. Bedair, Appl. Phys. Lett. 79, 3473 (2001); S. Sonoda, S. Shimizu, T. Sasaki, Y. Yamamoto and H. Hori, J. Cryst. Growth 237-239, 1358 (2002); T.
Sasaki, S. Sonoda, Y. Yamamoto, K. Suga, S. Shimizu, K. Kindo, and H. Hori, J. Appl. Phys. 91, 7911 (2002).

7 S. P. Guo, H. Ohno, A. Shen, F. Matsukura, and Y. Ohno, Appl. Surf. Sci. 130-132, 797 (1998).

${ }^{8}$ H. Ofuchi, T. Kubo, M. Tabuchi, Y. Takeda, F. Matsukura, S. P. Guo, A. Shen, and H. Ohno, J. Appl. Phys. 89, 66 (2001).

9 D. Loss and D. P. DiVincenzo, Phys. Rev. A 57, 120 (1998).

10 G. Burkard, H.-A. Engel, D. Loss, Fortschr. Phys. 48, 965 (2000).

11 A. Georges, G. Kotliar, W. Krauth, and M. J. Rozenberg, Rev. Mod. Phys. 68, 13 (1996).

12 A. Auerbach, Interacting Electrons and Quantum Magnetism (Springer, New York, 1994).

13 P. Lyu and K. Moon, Phys. Rev. B 64, 035201 (2001).

14 J. M. Kikkawa and D. D. Awschalom, Nature 397, 139 (1999); J. M. Kikkawa, J. A. Gupta, I. Malajovich, and D. D. Awschalom, Physica E 9, 194 (2001). 